\title{
In-Plane Orientation in Poly(amic acid)s during Thermal Imidization
}

\author{
Shin'ya MORINO ${ }^{\dagger}$ and Kazuyuki HORIE* \\ Photofunctional Chemistry Division, Research Laboratory of Resources Utilization, Tokyo \\ Institute of Technology, 4259 Nagatsuta, Midori-ku, Yokohama 226-8503, Japan \\ * Department of Chemistry and Biotechnology, Graduate School of Technology, \\ The University of Tokyo, 7--3-1 Hongo, Bunkyo-ku 113-8656, Japan
}

(Received March 5, 1999)

\begin{abstract}
Refractive indices of in-plane and out-of-plane directions were measured for a poly(amic acid) (PAA) synthesized from biphenyltetracarboxylic dianhydride and $p$-phenylenediamine and PAA synthesized from biphenyltetracarboxylic dianhydride and $p, p^{\prime}$-diaminodiphenylether. Depth dependence of in-plane orientation was observed for both PAAs. The degree of in-plane orientation is larger around the air/film interface than the film/substrate interface. Factors which contribute to increase in average refractive index are solvent evaporation, thermal imidization and dense packing during heating. Solvent evaporation is dominant for curing at temperatures below $100^{\circ} \mathrm{C}$, thermal imidization is dominant for curing at temperatures from $100^{\circ} \mathrm{C}$ to $200^{\circ} \mathrm{C}$ and change in packing becomes important for curing at temperatures above $200^{\circ} \mathrm{C}$.

KEY WORDS Polyimides / In-Plane Orientation / Birefringence / Thermal Imidization / $\mathrm{m}$-Line Method

/ Polymer Thin Films /
\end{abstract}

In recent years, in-plane orientation of polyimides (PIs) has been of interest because of large birefringence. ${ }^{1-7}$. This birefringence can be applied to optical devices such as waveplates, ${ }^{2}$ polarizers and so on. Effort was also made to apply this material for a compensator of liquid crystal displays. $^{3}$

The stiffness of PI main chain affects physical properties and in-plane orientation. ${ }^{2-6}$ This stiffness is governed by the chemical structure of polyimides. Degree of in-plane orientation depends on mismatch of thermal expansion coefficient. ${ }^{2}$ Biaxial drawing takes place when volume loss occurs during solvent evaporation and imidization. Polyimide films are surrounded by superstrates (air in typical cases) and substrates and are asymmetric along the out-of-plane direction. It is expected to observe asymmetry in optical properties along the out-of-plane direction.

Optical properties of poly(amic acid)s (PAA) and polyimides (PI) reflect density, chemical composition and degree of orientation. For example, charge transfer absorption is observed for some polyimides, which shows the packing structure of the polyimide main chain. Because the refractive index, one of optical properties, is measured with high accuracy (at least 4 digits), it could be a useful method to investigate changes in physical and chemical properties.

In the present paper, refractive index of in-plane and out-of-plane directions are measured during thermal imidization of some poly(amic acid)s. Depth dependence of refractive indices and degree of in-plane orientation are discussed.

\section{EXPERIMENTAL}

\section{Preparation of Poly(amic acid) Films}

Figure 1 shows the chemical structures of poly(amic acid)s used in this study. Poly(amic acid)s, PAA(BPDA/PDA), synthesized from biphenyltetracarboxylic dianhydride and $p$-phenylenediamine, and PAA(BPDA/

\footnotetext{
${ }^{\dagger}$ To whom correspondence should be addressed.
}

ODA) synthesized from biphenyltetracarboxylic dianhydride and $p, p^{\prime}$-diaminodiphenylether were prepared by the conventional manner as mixing equal molar of a dianhydride and a diamine in a suitable solvent such as $N, N^{\prime}$-dimethylacetoamide (DMAc). The mixtures were sealed and stirred for one day, reprecipitated from methanol, and dried at room temperature for one day. The resulting PAAs were dissolved into DMAc again and spincoated ( $500 \mathrm{rpm}$ for $30 \mathrm{~s}$ ) on fused silica substrates, and dried under vacuum at $50^{\circ} \mathrm{C}$ for one day. Film thickness was set at $c a .5 \mu \mathrm{m}$.

\section{Refractive Index Measurements}

Refractive index measurements were carried out by an $m$-line method. ${ }^{13-15}$ Figure 2 depicts the measurement system. Films on fused silica substrates work as slab waveguides. Waveguided modes consist of two orthogonal modes, TE and TM. TE modes have a parallel electric component to the film plane, and TM modes have a parallel magnetic component to the film plane. Both

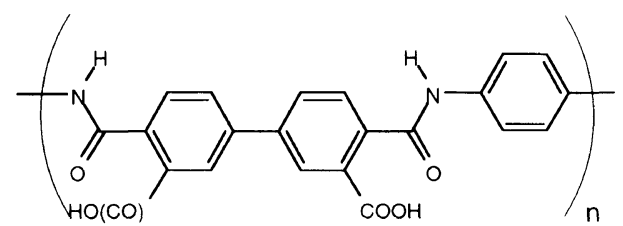

PAA(BPDA/PDA)

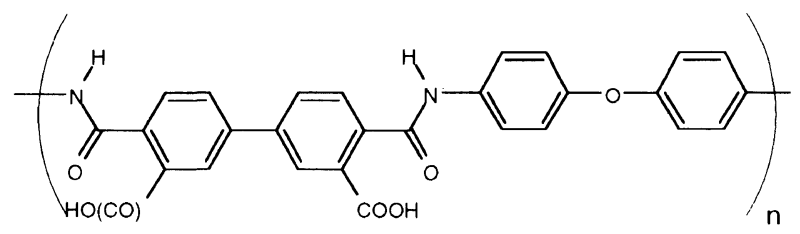

PAA(BPDA/ODA)

Figure 1. Chemical structures of poly(amic acid)s used in this study. 


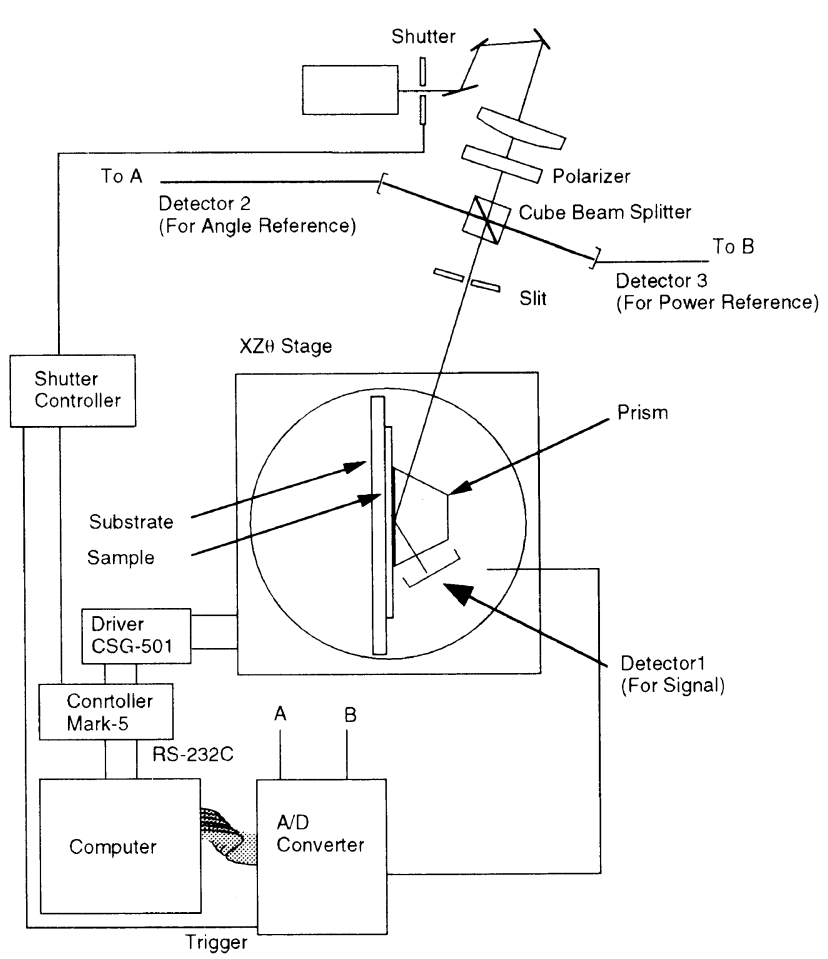

Figure 2. Setup for refractive index measurements by the $m$-line method.

modes are selected by rotating the plane of polarization of incident laser beam at $632.8 \mathrm{~nm}$ from a He-Ne laser. By using a prism coupler, waveguided modes were excited. Effective refractive indices were measured for each mode by measuring the incident angle to the prism plane when waveguided modes were excited most effectively. The intensity of waveguided light was determined with the intensity of scattering light from the waveguide. Effective refractive indices were calculated using eq 1 written as,

$$
n_{\text {eff }}=n_{\mathrm{p}} \sin \left(\alpha+\sin ^{-1}\left[\frac{n_{\text {air }}}{n_{\mathrm{p}}} \sin \left\{\theta_{\mathrm{obs}}\right\}\right]\right)
$$

where $n_{\text {eff }}$ is the effective refractive index, $n_{\mathrm{p}}, n_{\mathrm{air}}$, and $n_{\mathrm{film}}$ are refractive indices of the prism, the air, and films, respectively, and $\alpha$ is the angle of the prism, and $\theta_{\text {obs }}$ is the incident angle to the prism. The relationship between the effective refractive index, film thickness, $W$, and the refractive index of the film, $n_{\text {film }}$, are described by the following eigen value equation,

$$
\begin{gathered}
k_{0} W \sqrt{n_{\text {eff }}^{2}-n_{\text {film }}^{2}}-\Phi_{\text {sub }}-\Phi_{\text {air }}=m \pi \\
\Phi_{\mathrm{i}}=\arctan \left(\zeta\left(\frac{n_{\text {film }}}{n_{\mathrm{i}}}\right)^{a} \sqrt{\frac{n_{\text {eff }}^{2}-n_{\mathrm{i}}^{2}}{n_{\text {film }}^{2}-n_{\text {eff }}^{2}}}\right) \\
\mathrm{i}=\text { air, sub }
\end{gathered}
$$

where $k_{0}$ is the wavenumber of the incident laser beam in vacuum, an integer, $m$, is the mode number, and $n_{\text {sub }}$ is the refractive index of the substrate. $n_{\mathrm{TE}}$ and $n_{\mathrm{TM}}$ represent the refractive index of in-plane and out-of-plane orientation of films, respectively. For TE modes, $\zeta=1$, $a=0$. For TM modes, $\zeta=\left(n_{\mathrm{TE}} / n_{\mathrm{TM}}\right), a=2 . n_{\mathrm{TE}}, n_{\mathrm{TM}}$, and $W$ were calculated by least square fitting using eq 1,2 with appropriate values for $n_{\mathrm{p}}, n_{\mathrm{air}}, n_{\mathrm{sub}}$, and $\alpha$.
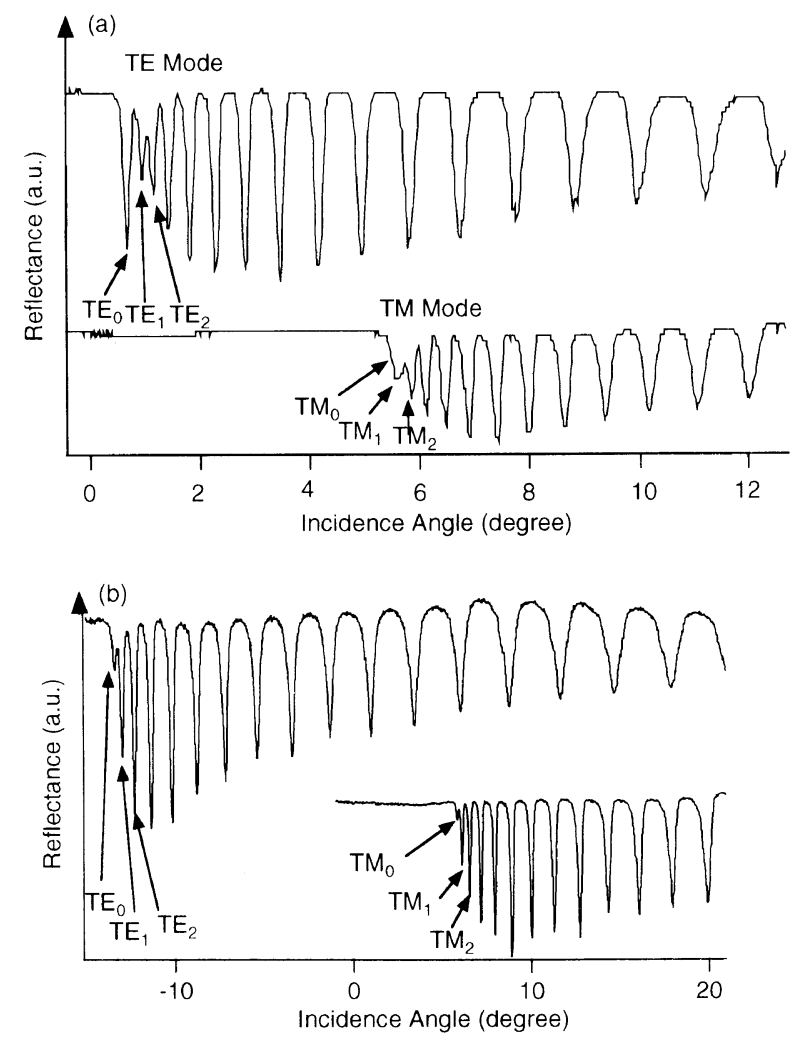

Figure 3. $m$-Line spectra of PAA(BPDA/PDA) before annealing (a) and after annealing at $160^{\circ} \mathrm{C}(\mathrm{b})$.

\section{Refractive Index Measurements During Stepwise Thermal Imidization}

Refractive indices were measured for PAA films of various degrees of thermal imidization by heating at temperatures from $50^{\circ} \mathrm{C}$ to $350^{\circ} \mathrm{C}$ for $2 \mathrm{~h}$ under vacuum successively. Refractive indices were measured after heating at each temperature.

\section{Determination of Degree of Imidization}

Free-standing PAA films fabricated in the same manner as above were used for IR spectra measurements. The degree of imidization was obtained from IR absorbance of imide carbonyl group at $1780 \mathrm{~cm}^{-1}$ with the internal standard of IR absorbance of a benzene ring at $1515 \mathrm{~cm}^{-1}$.

\section{RESULTS AND DISCUSSION}

Figure 3 shows the typical $m$-line spectra of PAA(BPDA/PDA) before annealing and after annealing at $160^{\circ} \mathrm{C}$. This result for the TE mode cannot be analyzed with the assumption of no depth dispersion of refractive index. As shown in Figure 3(a), $m$-line spectra of PAA(BPDA/PDA) show unusual behavior that the $\mathrm{TE}_{0}$ peak is stronger than $\mathrm{TE}_{1}$ peak, and the interval between $\mathrm{TE}_{0}$ and $\mathrm{TE}_{1}$ is wider than that between $\mathrm{TE}_{1}$ and $\mathrm{TE}_{2}$, though this behavior is not observed for TM modes. For step-index planar waveguides, coupling efficiency from a prism to waveguide, calculated from the dip in reflectance, is larger for modes of higher order because evanescent field is stronger for waveguided modes of higher order, and intervals between the neiboring modes are smaller for modes of lower order. Such behavior 


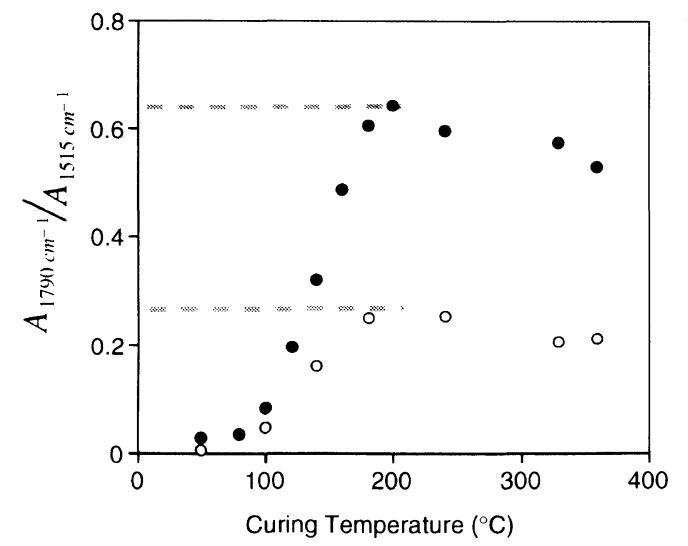

Figure 4. Curing temperature dependence of the conversion of imidization. Ratio of absorbance at $1790 \mathrm{~cm}^{-1}$ to that at $1515 \mathrm{~cm}^{-1}$ is plotted for PAA(BPDA/PDA) (O) and PAA(BPDA/ODA) (O).

was not observed for annealed PAA(BPDA/PDA) film as shown in Figure 3(b). Therefore annealed film can be treated as a step-index waveguide. Figure 3(a) suggests that there is strong depth dependence of refractive index, and is explained as follows. Refractive index of PAA films is larger around an air/waveguide interface, which results in total reflection of waveguided light in the middle of the film, and reduces the effective thickness for the $\mathrm{TE}_{0}$ mode. $\mathrm{TE}_{0}$ mode is guided around an air/waveguide interface, and intensity of evanescent field is stronger than the $\mathrm{TE}_{1}$ mode at the air/film interface, which increases coupling efficiency. $\mathrm{TM}_{0}$ modes for both films were very weak, which shows that this mode is wellguided, and intensity of evanescent wave is very weak. This suggests lower refractive index at the air/film interface than that at the film/substrate interface. From these results, we conclude that in-plane orientation proceeds faster around an air/film interface than film/ substrate interface. We had expected that strong orientation around film/substrate interface would arise from the anchoring of PAA molecules on substrates as shown in liquid crystal/substrate interfaces. But substrates do not play an important role except of binders of films.

To calculate refractive indices, the $\mathrm{TE}_{0}$ mode was ignored to eliminate the effect of the depth dependence of refractive index. Refractive indices were obtained as 1.651 for $n_{\mathrm{TE}}$ and 1.597 for $n_{\mathrm{TM}}$ of PAA(BPDA/PDA). Values were also 1.634 for $n_{\mathrm{TE}}$ and 1.602 for $n_{\mathrm{TM}}$ for PAA(BPDA/ODA). Film thickness was also obtained by $m$-line measurements as $c a$. $5 \mu \mathrm{m}$ for both films. Thickness dependence of in-plane-orientation has been investigated, and reported thinner than $10 \mu \mathrm{m}$ required for strong in-plane orientation. ${ }^{9,12}$ Both PAA films show birefringence between in-plane and out-of-plane directions. In-plane orientation of polyimides arises from biaxial drawing caused by volume-loss during imidization, ${ }^{1,2,6}$ which occurs because films are bounded to substrates. The origin of volume loss for PAA films is evaporation of a casting solvent. Evaporation of the solvent is faster around the air/film interface than around the film/substrate interface, which results in the depth dependence of in-plane orientation. The same behavior was observed for $m$-line spectra of PAA(BPDA/ODA).
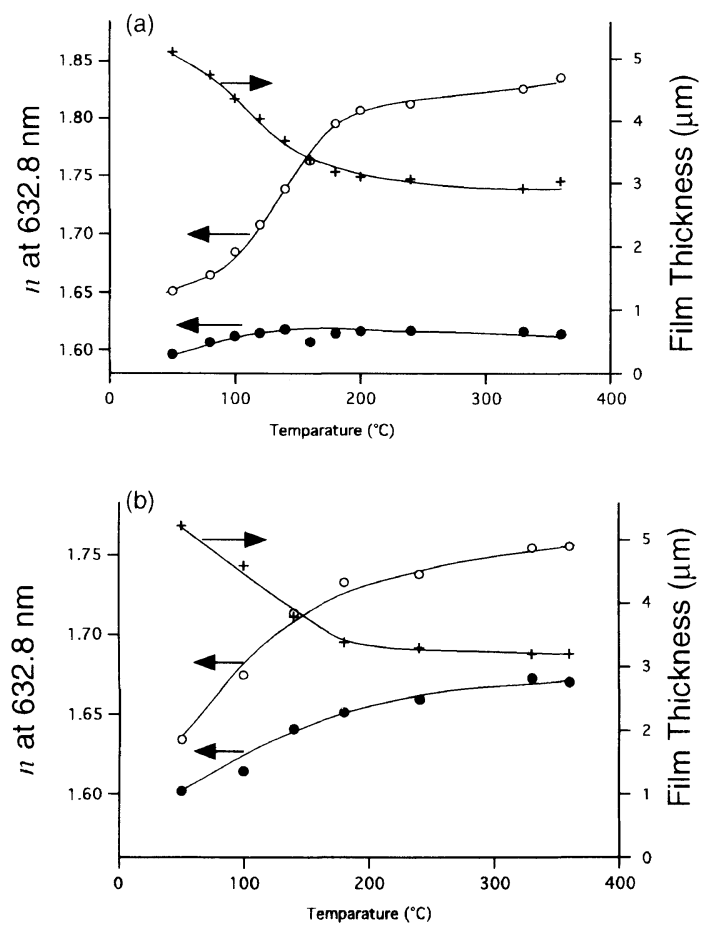

Figure 5. Curing temperature dependence of refractive indices of out-of-plane direction (O) and in-plane direction $(O)$, and film thickness (+) for PAA(BPDA/PDA) (a) and PAA(BPDA/ODA) (b).

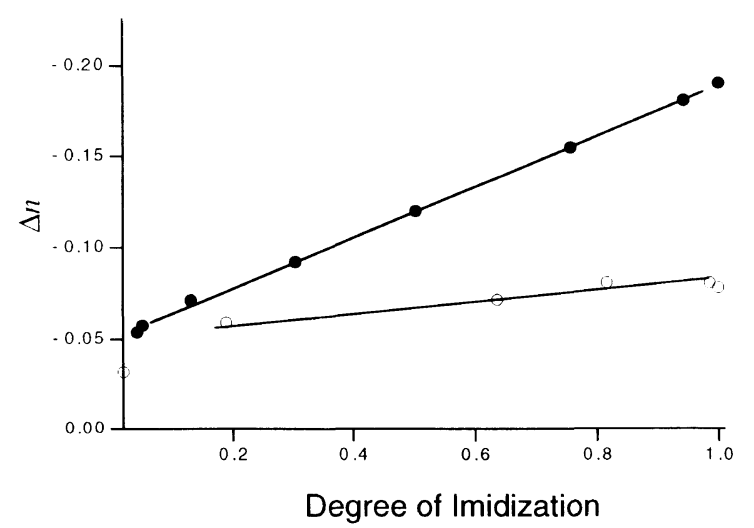

Figure 6. Birefringence as a function of imidization for PAA(BP$\mathrm{DA} / \mathrm{PDA})(\mathrm{)})$ and for PAA(BPDA/ODA) $(\bigcirc)$.

Figure 4 shows estimation of the degree of imidization. The ratio of absorbance at $1780 \mathrm{~cm}^{-1}$ to that at $1515 \mathrm{~cm}^{-1}$ saturated around $200^{\circ} \mathrm{C}$, caused by the fact that the absorption at $1780 \mathrm{~cm}^{-1}$ includes absorption which arises from acid anhydride whose formation was confirmed by IR absorption band at $1850 \mathrm{~cm}^{-1}$. Here we approximated that imidization finished at about $200^{\circ} \mathrm{C}$.

Figure 5 shows curing temperature dependence of $n_{\mathrm{TE}}$, $n_{\mathrm{TM}}$ and film thickness obtained by $m$-line measurements. Birefringence, $\Delta n=\left(n_{\mathrm{TM}}-n_{\mathrm{TE}}\right)$, calculated from the obtained values in Figure 5 is plotted as a function of the conversion of imidization in Figure 6. Values of birefringence are negative because of negative anisotropy of in-plane orientation. The birefringence of PAA(BPDA/PDA) shows linear relationship with the conversion of imidization, though that of PAA(BPDA/ODA) shows only slight increase with the conversion of imidization larger than 0.6. Similar behavior was reported for tensile 

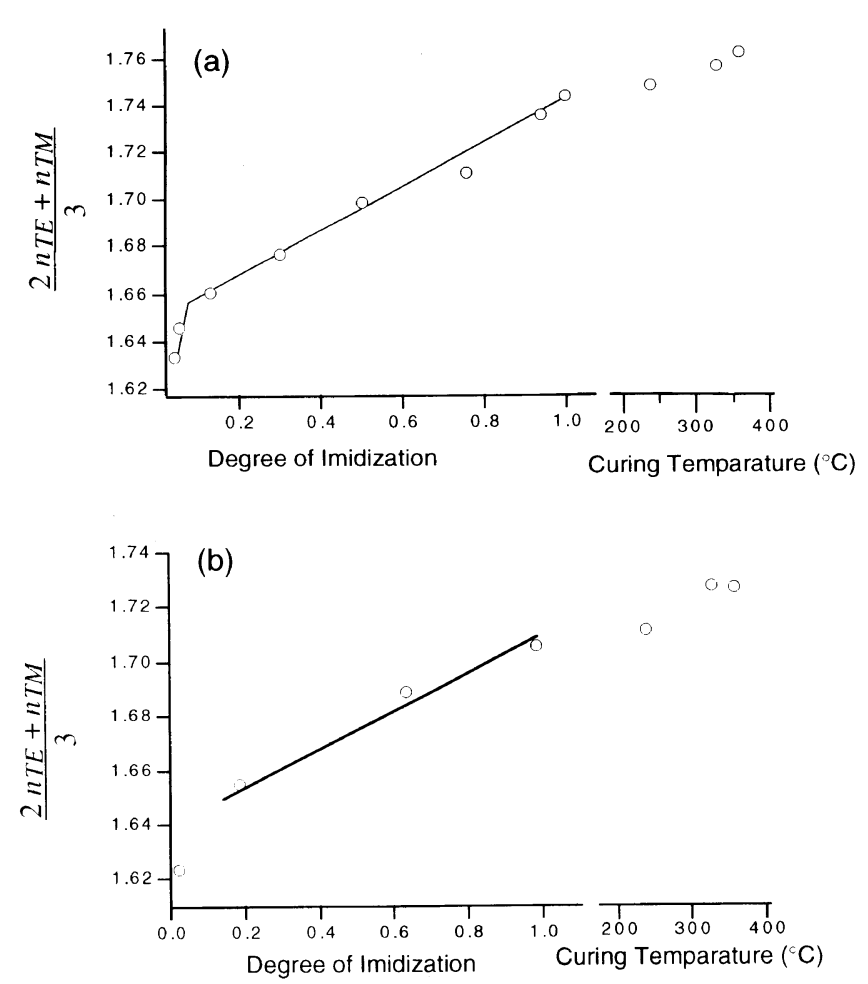

Figure 7. Conversion dependence of average refractive index for $\mathrm{PAA}(\mathrm{BPDA} / \mathrm{PDA})$ (a) and PAA(BPDA/ODA) (b).

strength on axial drawing of the same poly(amic acid)s. The bent in main chain at the ether group of ODA may thus cause sliding between polymer chains, which weakens the axial ordering of poly(amic acid)s.

The depth dependence of in-plane orientation observed for PAA films was not observed for films cured above $120^{\circ} \mathrm{C}$. Because thickness decreased and imidization proceeds all depth regions, the $\mathrm{TE}_{0}$ mode is not affected by the depth dependence of in-plane orientation.

For detailed analysis of refractive index and thickness changes, the average refractive index of $\left[\left(2 n_{\mathrm{TE}}+n_{\mathrm{TM}}\right) / 3\right]$ is plotted as a function of the degree of imidization in Figure 7. In the figure, the results for temperatures higher than $240^{\circ} \mathrm{C}$ are plotted as a function of curing temperature because conversion of imidization was assumed to be unity. For PAA(BPDA/PDA) rapid increase in average refractive index for degree of imidization from 0 to 0.05 , and slow increase for conversion of imidization larger than 0.05 were observed. According to the Lorentz-Lorenz equation, refractive index is determined by molar refraction and density. Molar refraction is affected by chemical composition, which corresponds to solvent content and degree of thermal imidization. Change in density was observed as thickness change. The rapid increase is induced by the evaporation of the casting solvent. This is dominant for curing below $100^{\circ} \mathrm{C}$ which corresponds to the degree of imidization of 0.05 . The slow increase is due to thermal imidization. Birefringence continued to increase for curing above $200^{\circ} \mathrm{C}$. This reflects increase in density because of dense packing of aromatic imide groups. Similar behavior was observed for PAA(BPDA/ODA).

\section{CONCLUSION}

Refractive indices of in-plane and out-of-plane directions were measured for poly(amic acid)s, PAA(BPDA/PDA) and PAA(BPDA/ODA). Depth dependence of in-plane orientation was observed for both PAAs. Degree of in-plane orientation is smaller around the film/substrate interface than around the air-film interface, though films are drawn because of being bound to substrates. Solvent evaporation, thermal imidization and dense packing contribute to increase in average refractive index during thermal imidization. Solvent evaporation is dominant for curing below $100^{\circ} \mathrm{C}$, thermal imidization from $100^{\circ} \mathrm{C}$ to $200^{\circ} \mathrm{C}$, and change in packing becomes important for curing above $200^{\circ} \mathrm{C}$.

Acknowledgments. This work is partly supported by a grant-in-aids for Scientific Research (06555287) from the Ministry of Education Science, Sports and Culture of Japan. The authors thank Dr. M. Hasegawa in Toho University for helpful discussion.

\section{REFERENCES}

1. T. P. Russel, H. Gugger, and J. D. Swalen, J. Polym. Sci., Polym. Phys. Ed., 21, 1745 (1983).

2. S. Ando, T. Sawada, and Y. Inoue, in "Polymeric materials for Microelectronic Applications," H. Ito, S. Tagawa, and K. Horie, Ed., ACS Symposium Series 579, American Chemical Society, Washington, D.C., 1994, p 283.

3. F. Li, F. W. Harris, and S. Z. D. Cheng, Polymer, 37, 5321 (1996).

4. R. M. Ikeda, J. Polym. Sci., Polym. Lett. Ed., 4, 353 (1996).

5. M. Hasegawa, T. Matano, Y. Shindo, and T. Sugimura, J. Photopolym. Sci. Technol., 7, 275 (1994).

6. M. Hasegawa, T. Matano, Y. Shindo, and T. Sugimura, Macromolecules, 29, 7987 (1996).

7. S. Ermer, in "Photosensitive Polyimides," K. Horie, and T. Yamashita, Ed., Technomic Publ. Co., Lancaster, 1995, Chapter 10.

8. L. Lin and S. A. Bidstrup, J. Appl. Polym. Sci., 54, 553 (1994).

9. M. Ree, Y.-H. Park, K. Kim, C. K. Cho, and C. E. Park, Polymer, 38, 6333 (1997).

10. D. Boese, H. Lee, D. Y. Yoon, J. D. Swalen, and L. F. Tabolt, J. Polym. Sci., Phys. Ed., 30, 1321 (1992).

11. M. Ree, C. Chu, and M. J. Goldberg, J. Appl. Phys., 75, 1410 (1994).

12. F. Li, K. Kim, E. P. Savitski, J. Chen, F. W. Harris, and S. X. D. Cheng, Polymer, 38, 3223 (1997).

13. P. K. Tien, Appl. Opt., 10, 2395 (1971).

14. W. M. Prest and D. J. Luca, J. Appl. Phys., 50, 6067 (1979).

15. W. M. Prest and D. J. Luca, J. Appl. Phys., 51, 5170 (1980). 